\title{
Combining Scanning Transmission Electron Microscopy and Electron Diffraction to Understand the Atomic Structures of Oxide Superlattices
}

\author{
A. B. Shah, ${ }^{* * *}$ B.B. Nelson-Cheeseman, ${ }^{* * *}$ A. Bhattacharya, ${ }^{* * *}$ J.-M. Zuo, ${ }^{* *}$ and J. C.H. Spence* \\ * Department of Physics, Arizona State University, Tempe, AZ 85287-1504 \\ ** Department of Materials Science and Engineering and Frederick Seitz Materials Research \\ Laboratory, University of Illinois at Urbana-Champaign, Urbana, IL 61801 \\ *** Center for Nanoscale Materials and Materials Science Division, Argonne National Laboratory, \\ Argonne, IL 60439
}

Progress in the oxide materials community has led to the development of emergent materials with novel properties such as conducting oxide interfaces and ferromagnetism, for example. Many of these materials are grown as epitaxial thin films on crystalline substrates via pulsed laser deposition (PLD) or molecular beam epitaxy (MBE). The magnitude of transport properties is highly dependent on the growth conditions, underlying substrate structure, strain, interface density, interfacial states, stoichiometry, and defect density. In the case of ultrathin films (less than $20 \mathrm{~nm}$ thick), the conventional array of ex-situ characterization techniques including x-ray diffraction and selected area electron diffraction (SAED) sample too large of an area to understand the localized structure, defects, and presence of interfacial states. Thus localized structural characterization from other techniques is highly desirable for thin films and nanostructures.

Nanoarea electron diffraction (NED) in the transmission electron microscope (TEM) has the ability to probe small features sizes from a few tens of $\mathrm{nm}$ to $100 \mathrm{~nm}$ without selection errors introduced by post specimen apertures in SAED. NED can take diffraction from a superlattice, but typically cannot select an individual film less than $10 \mathrm{~nm}$ thick. More recently, atomic resolution scanning transmission electron microscopy (STEM) can be used to image sub-nm structures such as interfaces, defect structures, and can show the extent of chemical intermixing and interdiffusion. HAADF STEM displays chemical contrast when the atomic number of the elements involved differ by Z. The increased resolution and stability of aberration corrected STEMs has allowed the use of fast Fourier transforms (FFTs) to extract localized structural information from portions of an image. In many cases, this allows one to obtain structural information from smaller areas than a NED probe can select. This can allow structural identification from very small areas comparable to the probe size in convergent beam electron diffraction, but with a more straightforward interpretation. The combination of STEM and NED is powerful for structural characterization of localized features such as individual thin films.

In addition, electron energy loss spectroscopy (EELS) acquired simultaneously with STEM can be used for chemical mapping and for detection of interfacial electronic states, such as valence and charge transfer. The advances in aberration correction and energy filters have dramatically improved the signal to noise ratio of EELS, and electron probes can be placed on individual interfaces for an understanding of new electronic states formed at the interface. This makes it possible to study electronic states as a function of interface quality. In this talk, I will discuss our structural and electronic characterization of several systems, including $\mathrm{MBE}$ grown $\mathrm{LaMnO}_{3}-\mathrm{SrMnO}_{3}$ and $\mathrm{La}_{1-\mathrm{x}} \mathrm{Sr}_{1+\mathrm{x}} \mathrm{MnO}_{4}$ superlattices. These superlattices show enhanced magnetization and electrical transport not found in bulk materials. The $\mathrm{LaMnO}_{3}-\mathrm{SrMnO}_{3}$ manganites show an enhanced magnetic 
response at the interfaces, depending on structural abruptness of the interfaces [1] [2]. The resistivity of the films is strongly dependent upon the supercell thickness. Electron diffraction and STEM results (Figure 1) show the presence of orthorhombic reflections in the larger period superlattices, which may be related to the magnetic and electrical transport characteristics. The $\mathrm{La}_{1-\mathrm{x}} \mathrm{Sr}_{1+\mathrm{x}} \mathrm{MnO}_{4}$ manganites are of the Ruddleson Popper structure and display an asymmetric magnetization in the in-plane and out-of-plane direction depending on the layering sequence [3]. Superlattices which have A-site ordering show a considerably larger magnetization out-of-plane over a randomly mixed alloy.

\section{References}

[1] S. May et al., Phys. Rev. B. 77 (2008) 174409.

[2] A. B. Shah et al., Phys. Rev. B. 82 (2010) 155112.

[3] B. B. Nelson-Cheeseman et al., Appl. Phys. Lett. In press. (2011).

[4] The work reported here are results of collaborations with research groups of A. Bhattacharya (Argonne National Laboratory), S. J. May (Drexel University) J. N. Eckstein (Physics, University of Illinois), Q. M. Ramasse (Lawrence Berkeley National Laboratory, SuperSTEM), and Jianguo Wen (Materials Research Laboratory, University of Illinois). The work is supported by DOE BES.
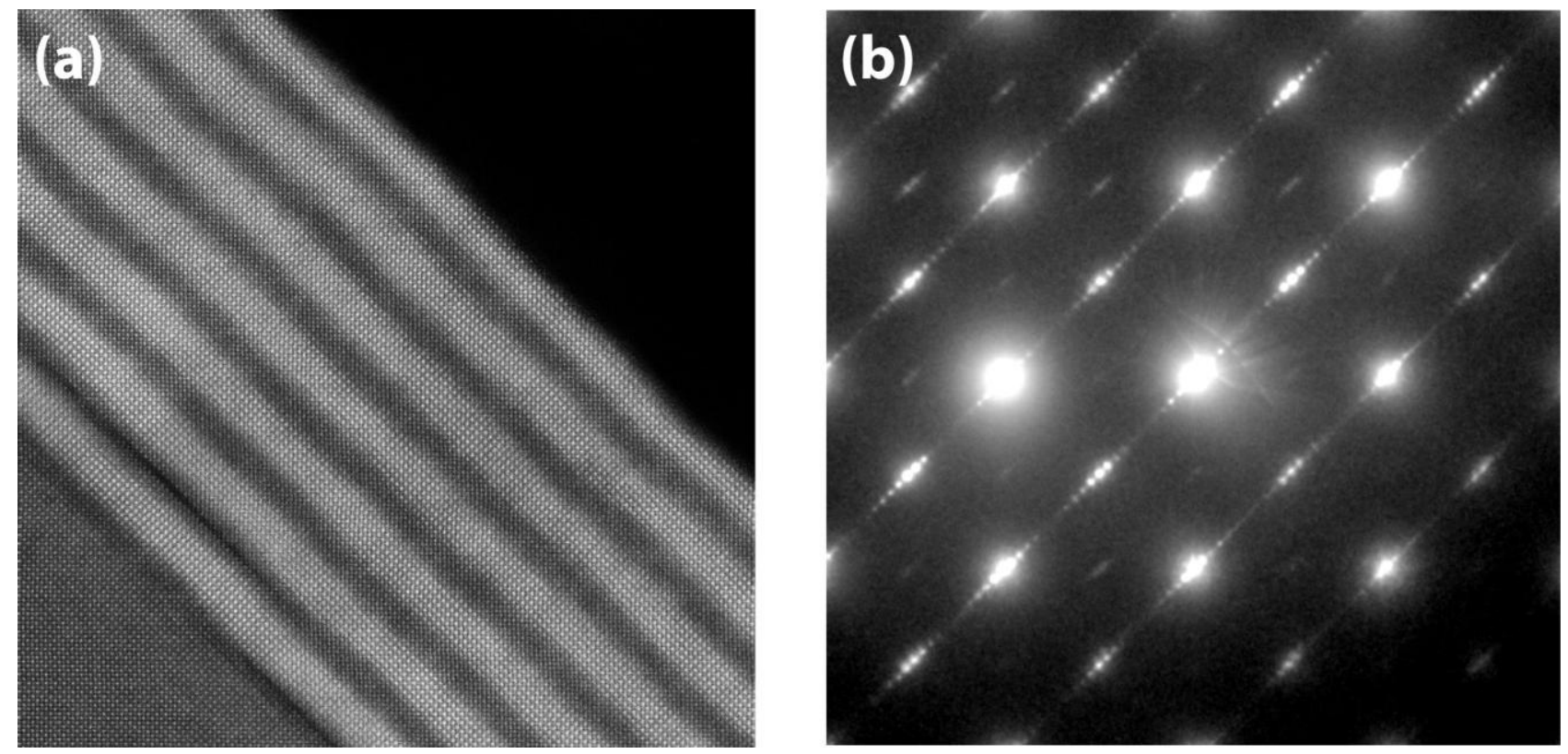

FIG. 1. (a) HAADF STEM image of a $\mathrm{LaMnO}_{3}-\mathrm{SrMnO}_{3}$ superlattice grown by MBE. The superlattice lattice parameter matches the underlying $\mathrm{SrTiO}_{3}$ in the in-plane direction and distorts in the out-of-plane direction without forming misfit dislocations. (b) Nanoarea electron diffraction pattern of the film shows superlattice reflections indicating high quality growth. There are also weaker reflections between Bragg reflections, which indicate the presence of an orthorhombic transformation. These orthorhombic reflections are only present in superlattices where the $\mathrm{LaMnO}_{3}$ films are thicker than 6 unit cells. 